FERMILAB-Conf-02/192-E

UASLP-IF-02-008

\title{
Two RICH Detectors as Velocity Spectrometers in the CKM Experiment*
}

J. Engelfried ${ }^{\mathrm{a} \dagger}$, P.S. Cooper ${ }^{\mathrm{b}}$, A. Morelos ${ }^{\mathrm{a}}$, I. Torres ${ }^{\mathrm{a}}$, A. Barker ${ }^{\mathrm{c}}$, L. Bellantoni ${ }^{\mathrm{b}}$, V. Bolotor ${ }^{\mathrm{d}}$, G. Britvich ${ }^{\mathrm{e}}$, M. Campbell ${ }^{\mathrm{f}}$, R. Coleman ${ }^{\mathrm{b}}$, C. Dukes ${ }^{\mathrm{g}}$, J. Frank ${ }^{\mathrm{h}}$, R. Gustafson ${ }^{\mathrm{f}}$, H. Huang ${ }^{\mathrm{c}}$, A.V. Inyakine, C.M. Jenkins ${ }^{\mathrm{i}}$, S.H. Kettell ${ }^{\mathrm{h}}$, T.R. Kobilarcik ${ }^{\mathrm{b}}$, V. Kurshetsov ${ }^{\mathrm{e}}$, A. Kushnirenko ${ }^{\mathrm{e}}$, L.G. Landsberg ${ }^{\mathrm{e}}$, K. Lang ${ }^{\mathrm{j}}$, S. Laptev ${ }^{\mathrm{d}}$, M. Longo ${ }^{\mathrm{f}}$, L. Lu ${ }^{\mathrm{g}}$, V. Molchanov ${ }^{\mathrm{e}}$, K. Nelsong ${ }^{\mathrm{g}}$, H. Nguyen $^{\mathrm{b}}$, R. Niclases ${ }^{\mathrm{c}}$, V. Obraztsov ${ }^{\mathrm{e}}$,H. Park ${ }^{\mathrm{f}}$, A. Pastsiak ${ }^{\mathrm{d}}$, S.I. Petrenko ${ }^{\mathrm{e}}$, A. Polarush ${ }^{\mathrm{d}}$, V. Polyakov ${ }^{\mathrm{e}}$, E. Ramberg ${ }^{\mathrm{b}}$, R. Strand ${ }^{\text {h, V.I. Rykalin }}{ }^{\mathrm{e}}$, R. Sirodeev ${ }^{\mathrm{d}}$, A. Soldatov ${ }^{\mathrm{e}}$, M.M. Shapkin ${ }^{\mathrm{e}}$, O.G. Tchikileve, R.S. Tschirhart ${ }^{\mathrm{b}}$, H.B. White ${ }^{\mathrm{b}}$, D. Vavilov ${ }^{\mathrm{e}}$, M. Wilking ${ }^{\mathrm{c}}$, J.Y. Wu ${ }^{\mathrm{b}}$, O. Yushchenko ${ }^{\mathrm{e}}$

anstituto de Física, Universidad Autónoma de San Luis Potosí, Mexico

${ }^{\mathrm{b}}$ Fermi National Accelerator Laboratory, Batavia, IL, USA

${ }^{\mathrm{c}}$ University of Colorado, Bolder, $\mathrm{CO}$

${ }^{\mathrm{d}}$ Institute of Nuclear Research, Troisk, Russia

${ }^{\mathrm{e} I n s t i t u t e}$ of High Energy Physics, Serpukhov, Russia

${ }^{\mathrm{f}}$ University of Michigan, Ann Arbor, Michigan 48109

'University of Virgina, Charlottesville, VA 22901

${ }^{\text {h}}$ Brookhaven National Laboratory, Upton, NY, USA

${ }^{\mathrm{i} U n i v e r s i t y ~ o f ~ S o u t h ~ A l a b a m a, ~ M o b i l e, ~ A l a b a m a ~} 36688$

${ }^{j}$ University of Texas at Austin, Austin, Texas 78712

We present the design of two velocity spectrometers, to be used in the recently approved CKM experiment. CKM's main goal is the measurement of the branching ratio of $K^{+} \rightarrow \pi^{+} \nu \bar{\nu}$ with a precision of $10 \%$, via decays in flight of the $K^{+}$. The design of both RICH detectors is based on the SELEX Phototube RICH. We will discuss the design and the expected performance, based on studies with SELEX data and Monte Carlo Simulations.

Keywords: RICH detector, Phototubes, rare kaon decay, ckm matrix

PACS: 29.40.Ka, 85.60.Ha, 13.20.Eb, 12.15.Hh

\section{The Physics}

The basic goal of the CKM experiment [6,7] is to determine $\left|V_{t d}\right|$, contributing to test the Standard Model hypothesis that a single phase in the

\footnotetext{
*Contributed talk at the Fourth Workshop on RICH Detectors, June 5-10, 2002, Pylos, Greece. To be published in NIMA.

${ }^{\dagger}$ Corresponding author; email: jurgen@ifisica.uaslp.mx
}

CKM matrix is the sole source of $\mathcal{C P}$ violation. The idea is to over-constrain the predictions with measurements. To really falsify the hypothesis, one needs a set of observables which can not only be measured sufficiently well, but also need to have controlled errors in the theoretical connection between the observable and the CKM matrix elements. A short list of these clean mea- 


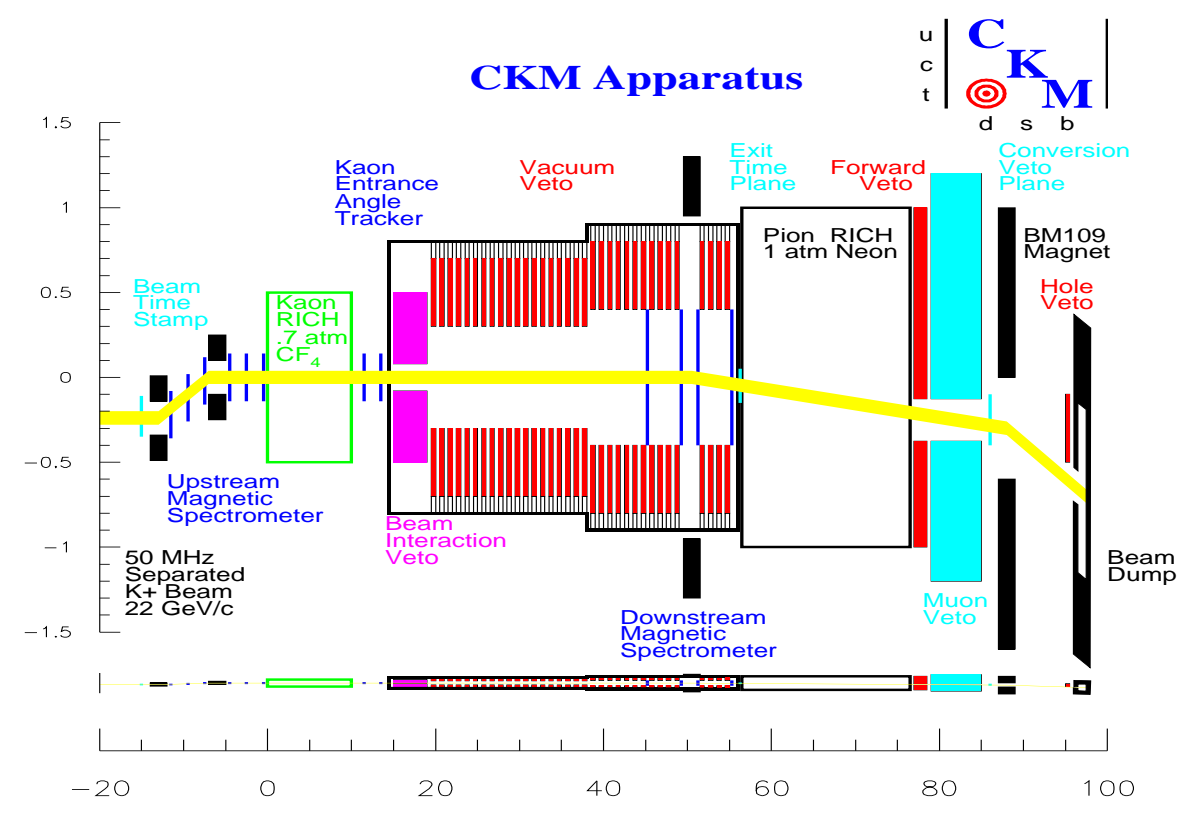

Figure 1. Proposed layout of the CKM experiment. Bottom: real scale. Top: Enlarged vertical scale.

surements to be made in the near future includes: $B_{d}^{0} \rightarrow \Psi K_{s}$ (the golden mode at the $B$-Factories), the ratio of the mixing parameters of $B_{d}$ and $B_{s}$ (to be measured at the Tevatron), $K^{+} \rightarrow \pi^{+} \nu \bar{\nu}$ and $K^{0} \rightarrow \pi^{0} \nu \bar{\nu}$.

The standard model prediction for the branching ratio of $K^{+} \rightarrow \pi^{+} \nu \bar{\nu}$ is [1,2] $0.55 \times$ $10^{-10}\left[(1.35-\bar{\rho})^{2}+(1.05 \bar{\eta})^{2}\right]$, defining an ellipse in the $\bar{\rho}-\bar{\eta}$ plane. The calculation [3] is done as a ratio to the branching ration of the decay $K^{+} \rightarrow \pi^{0} e^{+} \nu$, via weak isospin rotation. With current values this leads to $(0.72 \pm 0.21) \times 10^{-10}$. The theoretical uncertainty is estimated to be around $8 \%$ [1]:2], and dominated by the uncertainty in the charm quark mass. We therefore aim at observing 100 events of that decay, giving a measurement of $\left|V_{t d}\right|$ of $10 \%$ including theoretical errors.

Recently the Brookhaven experiment 787 observed [4] two events of this decay after several years of running; their branching ratio measurement is high but consistent with the prediction of the Standard Model. A continuation of that stopped kaon experiment, E949, hopes to see 510 events in the next couple of years.

\section{The Experiment}

The CKM (Charged Kaons at the Main Injector) Collaboration plans to measure the branching ratio of $K^{+} \rightarrow \pi^{+} \nu \bar{\nu}$ to a statistical precision of $10 \%$ by observing the decay in flight. After a lengthy process [5:6.7] the experiment obtained Stage 1 (physics) approval at Fermilab in June 2001. The experiment plans to run in parallel to the Collider Run 2.

Protons from the Fermilab Main Injector are used to produce an RF-separated beam, containing $30 \cdot 10^{6} \mathrm{~K}^{+}$per second, with a total flux of $<50 \cdot 10^{6} / \mathrm{s}$. A layout of the experiment is shown in fig. 11. The largest part of the experiment is a $50 \mathrm{~m}$ long vacuum decay volume, filled with photon counters to veto against (under others) the decay $K^{+} \rightarrow \pi^{+} \pi^{0}$, one of the main 


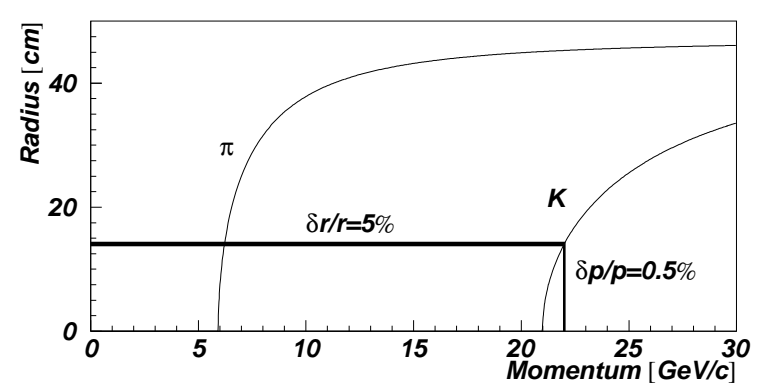

Figure 2. Ring Radius as a function of momentum in the Kaon RICH

backgrounds to suppress. This system should give a $\pi^{0}$ rejection of $<1.6 \cdot 10^{-7}$, requiring an additional kinematic rejection of $<3 \cdot 10^{-5}$ by redundant spectrometer systems: two conventional magnetic spectrometers and two velocity spectrometers based on RICH detectors. The kinematic rejection uses the fact that the main background modes are two-body decays, and, if calculating the invariant mass the $\pi^{+}$recoils to $\left(M_{\text {miss }}\right)$, results within resolution a fixed value, whereas the signal mode is a three-body decay, leading to a distribution in $M_{\text {miss }}$. The spectrometer systems have to measure the magnitude of the momenta of the $K^{+}$and $\pi^{+}$, as well as the decay angle between the $K^{+}$and $\pi^{+}$.

\section{RICHes as Velocity Spectrometers}

The velocity spectrometers consist of RICH detectors, which will measure the vector velocity (magnitude via the ring radius, track angles via the position of the ring center) for the incoming $K^{+}$and for the outgoing $\pi^{+}$. The detectors are modeled after the SELEX RICH [8,9,10,11], following the founding principle of the experiment that only proven detector technologies should be used to avoid surprises while searching for very rare decays. The results from the SELEX RICH are being used to study resolution effects and to tune the Monte Carlo simulation. In fig. 2 we show, when operating the RICH close to threshold, that one can obtain with a modest accuracy

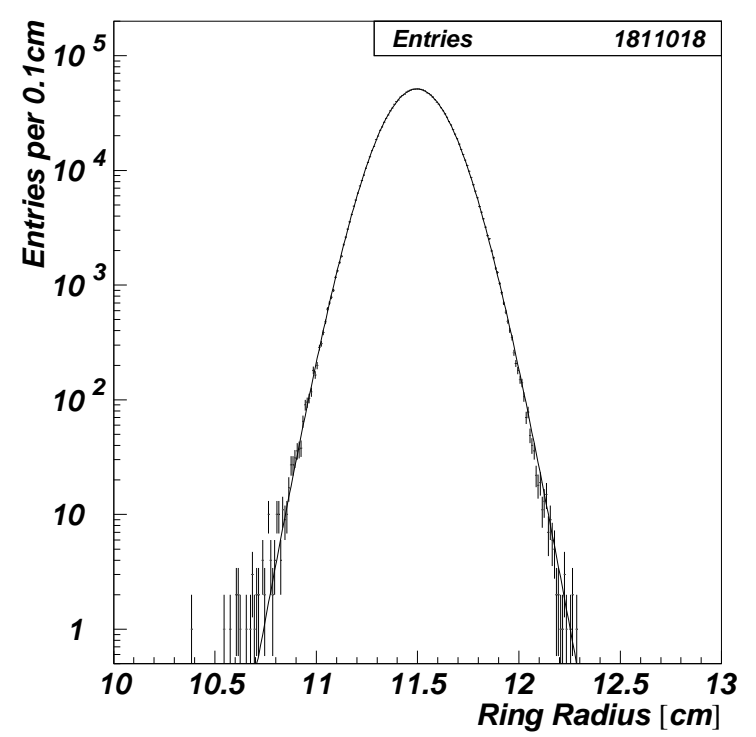

Figure 3. Ring radius distribution for $\beta=1$ particles in the SELEX RICH, using the standard SELEX algorithm. The curve fitted is explained in the text.

in ring radius an excellent measurement of momentum. In addition, the curves for kaon and pion are widely separated, so particle identification comes nearly for free. That a RICH can be operated in this regime was shown by the SELEX RICH 11.

The experiment requires momentum and angular resolutions in the order of $1 \%$, which can easily be translated into required ring radius resolutions. An even more important question is how gaussian is this resolution, e.g. how will nongaussian tails in the resolution function affect the overall performance when searching for rare decays. In fig. 3 we show the result of a study with SELEX data, single track events with $\beta=1$. To assign hits to a ring, we used the standard SELEX algorithm, which uses the information of the tracking system to determine the ring center. The average number of hits on a ring is 13.4 , with a Poisson distribution. We fit a sum of gaussians to 


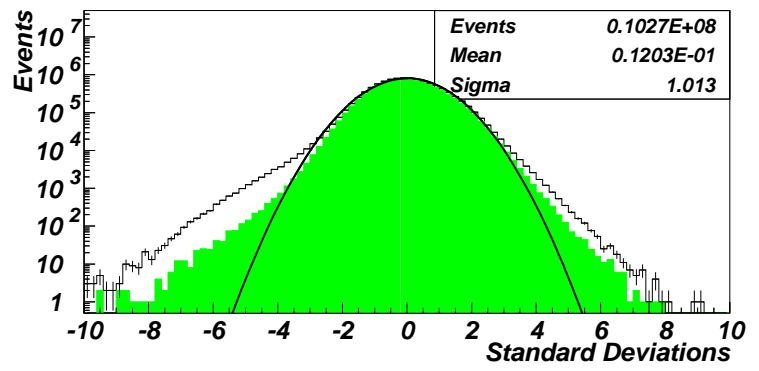

Figure 4. Pull distribution for the ring radius for $\beta=1$ particles in the SELEX RICH. A simple standalone pattern recognition is used. The two histograms show no and a soft cut.

this distribution, where the width of the gaussians is scaled by $\sqrt{N-3}$ ( $N$ being the number of hits on a ring), and the weight is given by how many events we observed for every given $N$. As can be seen, the resolution function shows a gaussian behavior over more than 4 orders of magnitude.

For CKM we do not want to use information from the tracking system in order to keep the two spectrometer systems really independent and redundant. We used the same SELEX sample as above, but this time performing a ring fit assigning all observed hits to the ring. We calculated the pull (deviation from mean divided by error) for the radius, where we scaled again the error with $\sqrt{N-3}$. The result is shown in fig. 1 . The gaussian behavior found in this study is already sufficient for the experiment. We are currently working on improving the pattern recognition algorithm. In addition, the integration in SELEX was $170 \mathrm{nsec}$, and in CKM we expect a time resolution in the order of a few nsec, which should help to reduce the noise contribution.

\section{The Pion RICH}

The Pion RICH will be a straight copy of the SELEX RICH, with the only exception of doubling the vessel length from $10 \mathrm{~m}$ to $20 \mathrm{~m}$, with a corresponding change in curva- ture of the mirrors to $40 \mathrm{~m}$. The radiator gas will be Neon at atmospheric pressure. Monte Carlo simulations showed that we should use $1 / 2$ in. photomultipliers sensitive down to $160 \mathrm{~nm}$ to obtain a momentum resolution in the $1 \%$ range. The resolution is balanced between the different contributions (pm size, chromatic dispersion), and the $\pi-\mu$ separation is $>10 \sigma$.

\section{The Kaon RICH}

Due to the beam characteristics (about $10 \mathrm{~cm}$ diameter, very small divergence) only a small number of phototubes is needed to cover the focal plane of the Kaon RICH. Due to space restrictions and rate problems, we will double-fold the light path in a $11 \mathrm{~m}$ long vessel, filled either with $\mathrm{N}_{2}$ at atmospheric pressure or with $\mathrm{CF}_{4}$ at about $0.7 \mathrm{~atm}$. The spherical mirror ( $40 \mathrm{~m}$ radius) and the second flat mirror will be outside the beam. The mirror sizes are chosen so that only part of the Cherenkov photons for the (bigger) pion ring are reflected, but the number of detected photons is still enough, as shown in fig. 5. The rings from kaons and pions are nicely separated, a fact we will use in the experiment trigger. The rates per tube will be as high as $1.4 \mathrm{MHz}$. The resolution is limited by chromatic dispersion, and we will use $1 / 2$ in. photomultipliers with a cutoff around $300 \mathrm{~nm}$.

\section{Resolution Studies with MC}

We implemented both RICHes, together with all other detectors, into GEANT 12]. We ran studies with up to $10^{6} \mathrm{~K}^{+} \rightarrow \pi^{+} \pi^{0}$ events with full kinematic reconstruction to verify the performance of single detectors and of the experiment as a whole. Detailed results can be found in [7]. A main result is that we have no correlations between the two spectrometer systems (magnetic, RICHes). With the current design we should be able to reach the goal of the experiment.

\section{Summary}

The recently approved CKM experiment will measure the branching ratio of $K^{+} \rightarrow \pi^{+} \nu \bar{\nu}$ with 100 events, to obtain a $10 \%$ measurement, includ- 

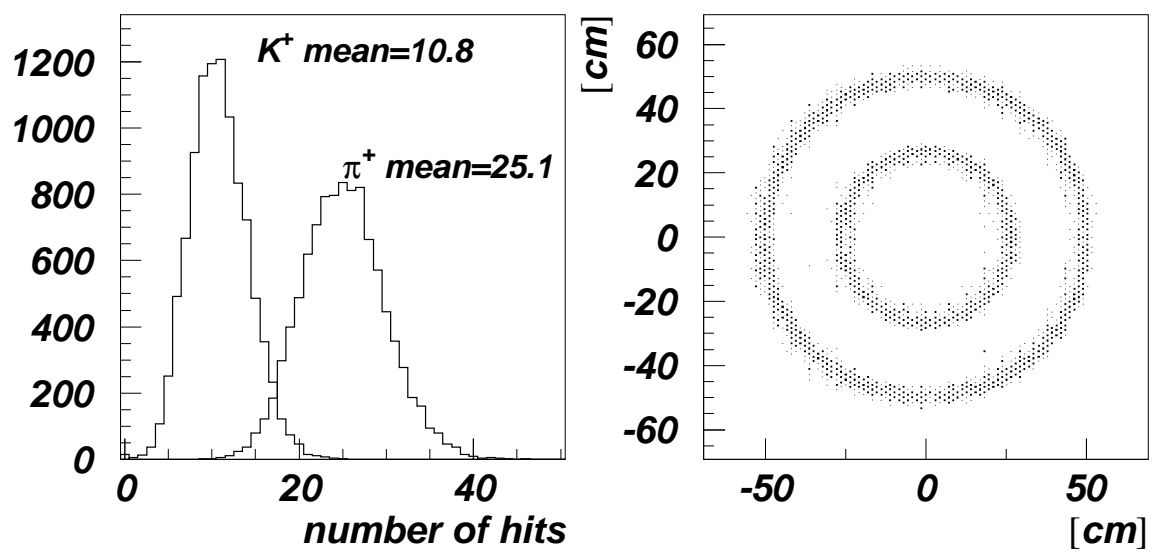

Figure 5. Results of Monte Carlo simulations for the Kaon RICH: Left: Number of photons detected for beam kaons and pions. Right: Distribution of detected photons in the focal plane for 10000 beam $K^{+}$ and 10000 beam $\pi^{+}$events.

ing theoretical uncertainties, of the magnitude of $V_{t d}$. We will employ two phototube RICH detectors, modeled after the SELEX RICH, as velocity spectrometers. We can stand high rates, have a very low noise rate, good time resolution (all intrinsic properties of photomultipliers), and we expect the non-gaussian tails of the resolution functions to be sufficiently small.

\section{Acknowledgments}

This work was supported in part by Consejo Nacional de Ciencia y Tecnología (Mexico), FAIUASLP, and the US Department of Energy (contract No. DE-AC02-76CHO3000 and DE-AC0298CH10886).

\section{REFERENCES}

1. A. Buras, in: Particles and Fields, Eighth Mexican Workshop, Zacatecas, Mexico, 2001. AIP Conference Proceedings 623 (Ed. DíazCruz, Engelfried, Kirchbach, Mondragón), pp. 3-35.

2. G. D'Ambrosio, G. Isidori, Phys. Letters B530 (2002) 108. hep-ph/0112135.
3. T. Inami and C.S. Lim, Progress of Theoretical Physics 65 (1981) 297-314.

4. S. Adler et al., Phys. Rev. Letters 88, 041803 (2002), hep-ex/0111091; S. Adler et al., Phys. Rev. Letters 84, 3768 (2000); S. Adler et al., Phys. Rev. Letters 79, 2204 (1997).

5. P.S. Cooper at al., Charged Kaons at the Main Injector - An experiment to measure $K^{+} \rightarrow \pi^{+} \nu \bar{\nu}$. Expression of Interest, April 1996.

6. CKM Collaboration, R. Coleman et al., CKM - Charged Kaons at the Main Injector - A proposal for a Precision Measurement of the Decay $K^{+} \rightarrow \pi^{+} \nu \bar{\nu}$ and Other Rare $K^{+}$Processes at Fermilab Using the Main Injector. Proposal FERMILAB-P-0905, April 1998.

7. CKM Collaboration, J. Frank et al., CKM - Charged Kaons at the Main Injector - A proposal for a Precision Measurement of the Decay $K^{+} \rightarrow \pi^{+} \nu \bar{\nu}$ and Other Rare $K^{+}$Processes at Fermilab Using the Main Injector. Proposal (2nd edition) FERMILAB-P-0921, April 2001.

8. J. Engelfried et al., Nucl. Instr. and Methods A409 (1998) 439. FERMILAB-Conf-97/210E. 
9. J. Engelfried et al., Nucl. Instr. and Methods A431 (1999) 53-69. hep-ex/9811001. FERMILAB-Pub-98/299-E.

10. J. Engelfried et al., Nucl. Instr. and Methods A433 (1999) 149-152. FERMILAB-Conf98/399-E.

11. J. Engelfried et al., Nuclear Instruments and Methods A, this volume. FERMILAB-Conf02/181-E.

12. GEANT - Detector Description and Simulation Tool, Version 3.21, CERN. 